Illinois State University

ISU ReD: Research and eData

Theses and Dissertations

$4-6-2021$

\title{
Handgrip Strength In Cancer Survivors: 2011-2014 National Health And Nutrition Examination Survey
}

Ayla Monet Kibler

Illinois State University, amkible@gmail.com

Follow this and additional works at: https://ir.library.illinoisstate.edu/etd

Part of the Physiology Commons

\section{Recommended Citation}

Kibler, Ayla Monet, "Handgrip Strength In Cancer Survivors: 2011-2014 National Health And Nutrition Examination Survey" (2021). Theses and Dissertations. 1382.

https://ir.library.illinoisstate.edu/etd/1382

This Thesis is brought to you for free and open access by ISU ReD: Research and eData. It has been accepted for inclusion in Theses and Dissertations by an authorized administrator of ISU ReD: Research and eData. For more information, please contact ISUReD@ilstu.edu. 


\title{
HANDGRIP STRENGTH IN CANCER SURVIVORS: 2011-2014 NATIONAL HEALTH AND NUTRITION EXAMINATION SURVEY
}

\author{
AYLA M. KIBLER
}

29 Pages

Cachexia, or a loss of muscle mass, has been identified as a potential indicator of cancer survival rates. Previous explorations in research have connected muscular strength to muscle loss and cancer survivorship, but nationally generalizable findings are limited. PURPOSE: To investigate handgrip strength in cancer survivors using the National Health and Nutrition Examination Survey (NHANES), a nationally representative sample of US adults. METHODS: The analysis included 368 males and 425 females from the 2011-2014 NHANES dataset. Cancer survivor information (type of cancer and age of diagnosis) was determined by questionnaire. Cancer types were grouped into the following: skin cancers, reproductive cancers, other cancers (digestive, nervous, respiratory, musculoskeletal, renal, and endocrine), and multiple or other cancers. Muscular strength was assessed using a handgrip dynamometer and expressed as relative grip strength z-scores. Low grip strength was categorized as $<33^{\text {rd }}$ percentile and sufficient strength was defined as relative grip strength at or above the $33^{\text {rd }}$ percentile. Logistic regression was used to estimate the odds of low relative grip-strength by cancer group and years from diagnosis, along with the following covariates: age, sex, minority status, social economic status (SES), body mass index (BMI), and physical activity (PA). A general linear model was also used to compare the effects of the previously mentioned cancer variables and covariates on continuous relative grip-strength z-scores. All analyses accounted for NHANES complex sampling, strata, weight, and clustering of data. RESULTS: In relation to relative grip strength, 
the cancer groups nor the years since diagnosis had a statistically significant impact on relative strength. Low relative grip strength was found only to be correlated with BMI $(\mathrm{p}<0.001)$ and PA $(\mathrm{p}=0.021)$. CONCLUSION: Cancer type and time since diagnosed with cancer were not found to be predictive of low relative grip strength. Other covariates, such as BMI and PA, were better predictors of low strength in cancer survivors. These results bring forth the importance of longitudinal analyses that look at strength before, during, and after a cancer diagnosis, in addition to details about the cancer diagnosis and treatments.

KEYWORDS: Cancer; Cachexia; Handgrip Strength; Body Mass Index; Physical Activity 
HANDGRIP STRENGTH IN CANCER SURVIVORS: 2011-2014 NATIONAL HEALTH AND NUTRITION EXAMINATION SURVEY

\author{
AYLA M. KIBLER
}

A Thesis Submitted in Partial Fulfillment of the Requirements for the Degree of MASTER OF SCIENCE

School of Kinesiology and Recreation

ILLINOIS STATE UNIVERSITY 
(C) 2021 Ayla M. Kibler 


\title{
HANDGRIP STRENGTH IN CANCER SURVIVORS: 2011-2014 NATIONAL HEALTH AND NUTRITION EXAMINATION SURVEY
}

\author{
AYLA M. KIBLER
}

COMMITTEE MEMBERS:

Kelly R. Laurson, Chair

Kristen M. Lagally

Dale Brown 


\section{ACKNOWLEDGMENTS}

First and foremost, I would like to thank my Father in Heaven who has provided many graces and blessings in order for me to complete my research successfully and with excellence. It is also a genuine pleasure to express my deepest gratitude to my thesis chair, Dr. Kelly Laurson, who displays an incredible passion for research and teaching. The guidance, mentorship, and all he has provided me throughout this experience is invaluable. It has been an honor to study under his lead. I also want to show my appreciation towards Dr. Kristin Lagally and Dr. Dale Brown, my committee members, for their time and assistance as we worked together to create something exemplary. This team has truly been a gift, and I would not have been able to do it without them. 


\section{CONTENTS}

Page

ACKNOWLEDGEMENTS

TABLES

$\begin{array}{ll}\text { CHAPTER I: INTRODUCTION } & 1\end{array}$

CHAPTER II: METHODS 3

$\begin{array}{ll}\text { Sample } & 3\end{array}$

Anthropometric Measures 3

$\begin{array}{ll}\text { Grip Strength } & 3\end{array}$

$\begin{array}{ll}\text { Cancer } & 4\end{array}$

$\begin{array}{ll}\text { Covariates } & 4\end{array}$

$\begin{array}{ll}\text { Statistical Analysis } & 5\end{array}$

$\begin{array}{lr}\text { CHAPTER III: RESULTS } & 6\end{array}$

$\begin{array}{lr}\text { CHAPTER IV: DISCUSSION } & 8\end{array}$

$\begin{array}{ll}\text { CHAPTER V: EXTENDED LITURATURE REVIEW } & 17\end{array}$

$\begin{array}{ll}\text { Summary } & 24\end{array}$

$\begin{array}{ll}\text { REFERENCES } & 26\end{array}$ 


\section{TABLES}

Table

Page

1. Descriptive statistics for cancer survivors by sex: NHANES 2011-2014

2. Prevalence of cancer types by sex: NHANES 2011-2014

3. Prediction of low relative grip strength by PA, BMI, cancer groups, and years since diagnosis

4. Percentage of cancer survivors with low or sufficient strength by years from cancer diagnosis

5. Mean relative grip strength z-scores by cancer type 


\section{CHAPTER I: INTRODUCTION}

In 2020, the National Cancer Institute estimated that by the end of the year there would be 1,806,590 new cases of cancer diagnosed in the United States and 606,520 people would die from the disease ${ }^{1}$. Many cancer patients, also known as cancer survivors, frequently experience an involuntary loss of weight, in particular loss of muscle mass, defined as cachexia ${ }^{2}$. In cancer survivors, any individual who has been diagnosed with cancer, cachexia is extremely prevalent, significantly reduces survival rates, and is the primary cause of death in one-quarter of the patients with advanced solid tumors ${ }^{3}$. Scientific understanding of cachexia has greatly improved in recent years, yet cancer cachexia still remains an unmet medical need and attempts towards a standard treatment guideline have been unsuccessful ${ }^{4}$.

Muscle strengthening may assist with the loss of muscle mass from cancer cachexia and may also being a predictor of cancer outcomes. One treatment used in an attempt to reverse the muscle/strength loss is exercise. Physical activity has proven to have a positive impact on cancer treatment related adverse effects ${ }^{5}$; patients who exercised, compared to patients who did not, experienced less negative effects and had a lower relative risk of cancer mortality and recurrence. In 2018, researchers ${ }^{6}$ used the National Health and Nutrition Examination Survey to assess 2773 individuals aged 50 or older. After adjusting for covariates, individuals in the upper quartile for muscle strength were at a $50 \%$ reduced risk of cancer specific mortality. There is also strong evidence for an association between reduced risk of bladder, breast, colon, endometrial, esophageal, renal, and gastric cancers and physical activity ${ }^{7}$. The same study also found a relationship between decreased all cause and cancer mortality and greater activity in individuals diagnosed with breast, colorectal, or prostate cancer, with up to $50 \%$ risk reduction. 
Unfortunately, few studies have described muscle strength across a diverse group of cancer survivors. This data would be helpful since different diagnoses and treatments of cancer have different effects on the survivors. In addition, cancer and muscle strength varies across different demographics, for example, age and sex. The literature has indicated links between strength and its impact on cancer survivorship, however, with studies being limited to small sample sizes and specific cancer types, there is a lack of nationally generalizable findings. Therefore, the purpose of this descriptive study is to explore handgrip strength in a large group of cancer survivors using the National Health and Nutrition Examination Survey (NHANES), a nationally representative sample. 


\section{CHAPTER II: METHODS}

\section{Sample}

Participants were from NHANES (2011-2014), a program used to assess the health and nutrition status of adults and children in the United States. This survey examines approximately 5,000 people each year via complex, multistage sampling. After processing inclusion criteria (full existing data of cancer diagnosis, grip strength, and other covariates), the final analytical sample included 793 subjects (unweighted), 368 males and 425 females. The National Center for Health Statistics (NCHS) reviewed and approved NHANES protocols. Informed consent was obtained from all participants.

\section{Anthropometric Measures}

Standing height was measured to the nearest $0.1 \mathrm{~cm}$ using a stadiometer (Seca, Hamburg, Germany) with a vertical backboard and headpiece. Body mass was measured to the nearest 0.1 $\mathrm{kg}$ on a digital weight scale (Mettler Toledo, Columbus, $\mathrm{OH})$. Body mass index (BMI) was

calculated as mass in kilograms divided by height in meters squared $\left(\mathrm{kg} / \mathrm{m}^{2}\right)$; subjects' BMI was categorized by underweight (>18.5), normal weight (18.5-24.9), overweight (25-29.9), or obese $(\geq 30)$.

\section{Grip Strength}

Muscular strength was measured via isometric grip strength using a handgrip dynamometer (Takei, Model T.K.K.5401, Niigata, Japan). A common way to assess muscular strength in this population is the handgrip test due to it being a feasible and valid predictor of total body muscular strength ${ }^{8}$. The hand dynamometer was adjusted to fit the participant's hand size. A practice trial was given to make sure the participant understood the procedure and that the grip size fit properly. Participants were tested standing with their elbow fully extended at the 
side without allowing the dynamometer to touch the body. Participants were asked to squeeze the dynamometer has hard as possible, making sure to exhale while squeezing. Each hand was tested three times, alternating between right and left, with a 60 second rest between measurements. The highest readings from each the left and right hand were taken and summed together for combined grip strength. Relative grip strength was then calculated by taking the participant's combined grip strength and dividing it by body mass (kg strength/ kg mass). An LMS growth model was used to calculate Z-scores from ages 20-80 years old; these relative Z-scores allow for standardization of grip strength by sex and age.

\section{Cancer}

Cancer was assessed in NHANES using a questionnaire which asks, "Ever told (by a doctor) you have cancer or malignancy?" to which subjects may reply, "Yes", "No, "Don’t Know". Then, participants were asked to list the types of cancer they have been diagnosed with, up to three types. The categories include bladder, blood, bone, brain, breast, cervix, colon, esophagus, gallbladder, kidney, larynx, leukemia, liver, lung, lymphoma, melanoma, mouth/tongue/lip, nervous system, ovary, pancreas, prostate, rectum, skin (non-melanoma), skin (unknown), soft tissue, stomach, testis, thyroid, uterus, other, or more than 3 kinds. This list was narrowed down and grouped into common subcategories: skin, reproductive, other cancers, or those reported as "multiple" or "other". NHANES also recorded the age when the cancer was first diagnosed. From this, an age difference variable was created by subtracting current age from the age of diagnosis. This allowed us to see how recently the diagnosis occurred.

\section{Covariates}

All analyses controlled for age, sex, race/ethnicity, physical activity, and socioeconomic status. The categories for race/ethnicity were Mexican American, other Hispanic, non-Hispanic 
white, non-Hispanic black, and other race/multiracial. Race from this population was primarily non-Hispanic white; therefore, two groups were made: non-Hispanic white and minority groups. Physical activity was based on a questionnaire given to the subjects asking how many minutes of activity they achieve per week at a moderate vigorous intensity. The participants reported minutes of vigorous-intensity work, minutes of moderate-intensity work, minutes walking or bicycling for transportation, minutes of vigorous recreation activities, and minutes of moderate recreation activities. These answers were summed into total minutes of moderate to vigorous physical activity per week. Socioeconomic status was measured by calculating the subjects' family income to poverty level ratio (FIPR). This was accomplished by dividing family income by the poverty guidelines, specific to family size, appropriate for the geographic location in the year of data collection.

\section{Statistical Analysis}

Descriptive statistics were calculated for individuals previously diagnosed with cancer, and each covariate separated by sex. A logistic regression was used to determine the odds of low relative grip-strength Z-scores (low strength $<33^{\text {rd }}$ percentile vs. sufficient strength $\geq 33^{\text {rd }}$ percentile) with the following covariates: sex, minority status, social economic status, BMI group, and physical activity. This logistic regression also sought to examine the differences between the cancer type subcategories. A general linear model was also used to compare the effects of the previously mentioned covariates on relative grip-strength Z-scores, with the additional covariate of years since the subjects' diagnosis. All of the above analyses accounted for NHANES' complex sampling, including the strata, the weight, and the clustering of the data. 


\section{CHAPTER III: RESULTS}

Descriptive statistics for male and female cancer survivors are displayed in Table 1 . The total sample was comprised of $87 \%$ individuals who categorized themselves as non-Hispanic white with an average FIPR score of $3.3(\mathrm{SD})$. The average age was $62(\mathrm{SD})$ years and the average number of years since their most recent cancer diagnosis was 10 (SD) years. The prevalence of various cancer types in males and females are presented in Table 2. For males, the top two cancer types were integumentary/skin cancer at $47.9 \%(3.1)$ and reproductive at $18.6 \%$ (2.4). For females, the top two cancer types were reproductive $40.3 \%$ (3.1) and integumentary/skin at $28.8 \%(2.9)$.

A general linear model (ANOVA) was also used to compare the effects of types of cancer groups and number of years since the subjects' cancer diagnosis, along with sex, minority status, social economic status, body mass index, and physical activity on relative grip-strength Z-scores. As displayed in Table 3, body mass index and physical activity were statistically significant predictors of low relative grip strength. According to the results, individuals who are overweight are 7.66 times more likely to have low relative grip strength, and individuals who are obese are 48.04 times more likely $(\mathrm{p}<0.001)$. Participants who recorded 0 minutes of physical activity were 2.17 times more likely to have low relative grip strength compared to individuals who meet the recommended physical activity of $150 \mathrm{~min} /$ week, and those who engage in 1-149 minutes of physical activity were 1.64 times more likely to have low relative grip strength $(p=0.021)$. Individuals diagnosed with reproductive cancer were 1.97 times more likely than those with skin cancer to have low strength scores; digestive, nervous, respiratory, musculoskeletal, renal, and endocrine cancer diagnoses were 1.23 times more likely than those with skin cancer; those with multiple cancers or recorded "other" were 2.05 times more likely than skin cancers to have low 
hand grip strength $(\mathrm{p}=0.074)$. Individuals who were diagnosed within 3 years or less were 1.75 times more likely to have low strength scores compared to those who were diagnosed 4 or more years ago $(\mathrm{p}=0.068)$. However, both the cancer groups and diagnosis within 3 years were not found to be statistically significant.

A logistic regression was used to determine the odds of low relative grip-strength Zscores (low strength $<33^{\text {rd }}$ percentile vs. sufficient strength $\geq 33^{\text {rd }}$ percentile) with the previously mentioned covariates. This logistic regression also sought to examine the differences between the cancer subcategories and the years from cancer diagnosis. Relative grip strength scores categorized by years since the subject's first cancer diagnosis can be found in Table 4. For individuals in the lower $33^{\text {rd }}$ percentile for strength scores, $21.7 \%$ was made up of patient's whose diagnoses were within 0 to 1 year, those within 2 to 5 years constituted $24.2 \%$, and last, those 6 or more years away from their first cancer diagnosis made up the remaining 54.1\%. Sufficient strength included $17.2 \%$ individuals 0 to 1 year from their diagnosis, 2 to 5 years also was $19.9 \%$, and finally 6 or more years since diagnosis made up $57.5 \%$. From these results, there was no relationship between the length in years from the participants diagnosis and their relative grip strength. Table 5 shows us the mean of relative grip strength z-scores for each cancer group. Skin cancer, reproductive cancers, digestive, nervous, respiratory, musculoskeletal, renal and endocrine cancers, and those with other or multiple cancers all had a mean score between 0.410.55 ; the z-scores being close to zero (the median) indicate that they are almost identical to the larger sample the z-scores were derived from. None of the four groups statistically different. 


\section{CHAPTER IV: DISCUSSION}

The goal of this descriptive study was to investigate handgrip strength in cancer survivors using the National Health and Nutrition Examination Survey (NHANES), a nationally representative sample. This study aimed to look at relative hand grip strength z-scores in individuals diagnosed with various types of cancer over many years, alongside covariates such as age, sex, minority status, social economic status, BMI, and physical activity.

Prior studies have shown that many individuals diagnosed with cancer suffer from a loss of muscle mass 91011 . This negatively affects patients' quality of life and cancer survival ${ }^{4} 12$ 135/20/2021 4:16:00 PM. Previous research has also shown that increased strength and exercise can have a positive impact for those diagnosed with cancer ${ }^{5}$. However, we found little evidence that different cancer types were associated with relative grip strength. When examining the odds ratio between skin cancers and all other types of cancer, all groups ranged between 1 to 2 times more likely to have low grip strength, and none were found to be statistically different $(\mathrm{p}=0.074)$. Individuals who were diagnosed within 3 years or less were 1.75 times more likely to score lower for strength, but this was not statistically significant either. The association between muscular strength and the number of years since each patient's first diagnosis also was not found to have any statistical significance in this study $(\mathrm{p}=0.068)$. Therefore, the cancer variables we examined, cancer type or years since diagnosis, did not have an association with relative grip strength.

From the literature, those who have higher strength are more likely to survive cancer ${ }^{7}$. While the results of this study did not replicate those findings directly, we did find that those who have higher PA $(\mathrm{p}=0.021)$ and lower BMI $(\mathrm{p}<0.001)$ are more likely to have higher relative strength. Cancer survivors whose lifestyles lack activity, reporting no moderate to vigorous PA, 
were over 2 times more likely to have low relative grip strength. Likewise, a cancer survivor classified as obese had an increased odds of low relative grip strength by almost 50 times over normal weight survivors. The significant relationships between PA and BMI with strength, highlights the importance of PA and BMI on survival.

In addition to PA and BMI, there were other findings from this study consistent with previous research. The family monthly poverty level index from NHANES, which is calculated by dividing family income by the poverty guidelines specific to family size, year, and state, range from 1 to 5 . The population in this study averaged a ratio of 3.6 for males, and 3.0 for females, showing that many of these cancer survivors are well above the poverty level. Previous research shows that in comparison to individuals with higher annual incomes, those with lower incomes have been found to have shorter survival times and lower survival rates ${ }^{14}{ }^{15}$. For this study's cancer survivors, the average number of years since their most recent diagnosis was about 10 years. These findings are coherent with research that shows higher income is related to greater survival length and rates. Similarly in our findings, we categorized SES into two groups, low SES and high SES, with a score of 2.28 as the cutoff. The low SES group had a mean of 0.44 , and the high SES group has a mean of 0.56 , for relative grip strength z-scores, with the differences being statistically significant. This indicates that individuals with a higher SES will also tend to score higher in grip strength, whereas those with low SES will score lower in grip strength.

Previous research has also found that cancer patients who exercise have a lower relative risk of cancer mortality and recurrence ${ }^{5}$. We explored grip strength z-scores by the number of years since the patients' diagnosis. However, years since diagnosis were not predictive of low strength: those scoring in the lower $33^{\text {rd }}$ percentile, or sufficient strength: those at or above the 
$33^{\text {rd }}$ percentile. In this sample, the largest proportion of survivors were 6 or more years away from their diagnosis. Knowing that many cancers are most likely to reoccur within the first 2 years, and after 5 years likelihood of recurrence is even less, it is likely that our population is relatively healthy considering more than half the population was already 6 or more years from their diagnoses ${ }^{16}$. This also would give the subjects years to regain their strength and resume their daily activities, which gives insight into the relative grip strength z-scores being analytically insignificant. After the 6 or more years category, 1 year from diagnosis was the next highest category for the low strength group. If the subjects' cancer is currently being treated, previous research shows this would impact their strength ${ }^{9}$. However, the other years are inconsistent, and the population with sufficient strength is evenly spread throughout the years, thus we are not able to conclude these findings as statistically significant.

The relative grip strength z-scores allows us to compare each individual's strength scores with others of the same demographic. Skin cancer averaged closest to 0 with a mean of 0.55 ; reproductive, digestive, nervous, respiratory, musculoskeletal, renal and endocrine cancers had a slightly lower mean of 0.49 ; those with other or multiple cancers had the lowest mean score of 0.41. All of these z-scores are near the median of the full NHANES dataset the z-scores were derived from. In addition to this, none of the four groups were statistically different. This demonstrates that our cancer survivor population is very similar in strength to our larger healthy population and cancer type was not a significant predictor of strength.

Prior research indicates that for every 100,000 people, the number of individuals newly diagnosed with cancer are 452 White, 440 Black, and 348 Hispanic; cancer death rates for every 100,000 cases are 156 White, 177 Black, and 110 Hispanic $^{17}$. We can see from these previous findings that the number of new diagnoses and the number of death rates do not have a large 
disparity between White and all other minority groups. However, the cancer survivors in our sample were composed of $87 \%$ individuals who classified themselves as Non-Hispanic White, and $13 \%$ classified as Mexican America, Non-Hispanic Black, Multiracial or other. This sample shows a much larger disparity between those who identify as White and those who identify with another race/ethnicity. Likewise, the mean relative grip strength z-scores for Non-Hispanic White compared to the minority group were not found to be statistically significant.

This study had many strengths, including the use of a national representative dataset that is generalizable to the noninstitutionalized US population. The data collection procedures were carried out by certified technicians with standardized quality control procedures. However, this study also had limitations, including over 30 cancer types, splitting the sample into many small groups. NHANES data collection is also cross-sectional and was limited in their information on cancer specifics (no inclusion of treatment, stage, current status, etc.).

In conclusion, this study was designed to look at relative grip strength z-scores in patients diagnosed over many years with various types of cancer, including age, sex, minority status, SES, BMI, and PA. Those who participated in more PA, had a lower BMI, and had higher SES, were more likely to have higher grip strength z-scores. However, we did not find evidence for cancer types or years since diagnosis to be predictive of low grip strength. It is likely that this cancer survivor population is similar in health to the larger NHANES population. Future longitudinal research is needed to look at grip strength from the moment of diagnosis, during treatment, and after survival, in order to analyze grip strength differences over time in individuals with cancer. Further studies should also explore grip strength in individuals receiving different cancer treatments, grip strength in cancer patients with cachexia compared to those without, and grip strength in relation to cancer stage. 
Table 1. Descriptive statistics for cancer survivors by sex: NHANES 2011-2014

\begin{tabular}{|c|c|c|}
\hline Variable & Males $(n=368)$ & Females $(n=425)$ \\
\hline Age (years) & $64.09(0.85)$ & $60.50(0.89)$ \\
\hline FIPR & $3.61(0.10)$ & $2.99(0.11)$ \\
\hline Height $(\mathrm{cm})$ & $176.03(0.52)$ & $161.77(0.48)$ \\
\hline Weight $(\mathrm{kg})$ & $88.84(1.19)$ & $76.88(1.30)$ \\
\hline $\mathrm{BMI}\left(\mathrm{kg} / \mathrm{m}^{2}\right)$ & $28.62(0.36)$ & $29.37(0.45)$ \\
\hline Minutes of MVPA per week & $520.1(46.7)$ & $409.2(40.0)$ \\
\hline Relative Combined Grip Z-score & $0.08(0.07)$ & $-0.0769(0.60)$ \\
\hline Age of most recent cancer diagnosis & $54.97(1.15)$ & $50.11(0.96)$ \\
\hline Years since most recent diagnosis & $9.11(0.68)$ & $10.39(0.61)$ \\
\hline \multicolumn{3}{|l|}{ Race } \\
\hline Mexican American & $1.3 \%(0.6)$ & $2.9 \%(1.0)$ \\
\hline Other Hispanic & $2.1 \%(0.7)$ & $3.2 \%(0.7)$ \\
\hline Non-Hispanic White & $88.4 \%(1.7)$ & $86.3 \%(2.2)$ \\
\hline Non-Hispanic Black & $5.5 \%(1.0)$ & $4.7 \%(1.0)$ \\
\hline Other/Multiracial & $2.5 \%(0.9)$ & $3 \%(1.5)$ \\
\hline \multicolumn{3}{|l|}{ BMI Category } \\
\hline Underweight & $0.1 \%(0.1)$ & $2 \%(0.8)$ \\
\hline Normal Weight & $25.6 \%(2.9)$ & $29.2 \%(2.6)$ \\
\hline Overweight & $40 \%(2.7)$ & $29 \%(3.4)$ \\
\hline Obese & $33.4 \%(2.7)$ & $39.8 \%(3.5)$ \\
\hline
\end{tabular}


Table 2. Prevelance of cancer types by sex: NHANES 2011-2014

\begin{tabular}{ccc}
\hline Variable & Males $(\mathrm{n}=368)$ & Females $(\mathrm{n}=425)$ \\
\hline Cancer Type & & \\
Digestive & $6.8 \%(1.5)$ & $4.8 \%(1.1)$ \\
Nervous & $0.4 \%(0.4)$ & $0.5 \%(0.3)$ \\
Integumentary & $47.9 \%(3.1)$ & $28.8 \%(2.9)$ \\
Respiratory & $3.7 \%(1.3)$ & $0.8 \%(0.4)$ \\
Musculoskeletal & $1.9 \%(0.9)$ & $1.1 \%(0.8)$ \\
Reproductive & $18.6 \%(2.4)$ & $40.3 \%(3.1)$ \\
Renal & $3.3 \%(1.1)$ & $1.4 \%(0.4)$ \\
Endocrine & $2 \%(0.9)$ & $6.2 \%(1.3)$ \\
Other & $4.2 \%(1.4)$ & $5.9 \%(1.2)$ \\
Multiple & $11.2 \%(1.8)$ & $10.3 \%(2.0)$ \\
\hline
\end{tabular}


Table 3. Prediction of low relative grip strength by PA, BMI, cancer groups, and years since diagnosis

\begin{tabular}{cc}
\hline Variable & Odds Ratio \\
\hline Physical Activity & $2.17(1.26-3.75)$ \\
$1-149$ min vs $150+$ min* $^{*}$ & $1.64(0.79-3.41)$ \\
Body Mass Index & $7.66(3.79-15.48)$ \\
Overweight vs Normal* & $48.04(26.22-88.01)$ \\
Obese vs Normal* & $1.97(1.06-3.67)$ \\
Cancer Groups & $1.23(0.57-2.62)$ \\
Reproductive Cancers vs Skin Cancers* & \\
Digestive, Nervous, Respiratory, & \\
Musculoskeletal, Renal, and Endocrine & vs Skin Cancers \\
Other and Multiple vs Skin Cancers & $2.05(0.95-4.43)$ \\
3 or less years vs 4 or more years & $1.75(0.94-3.25)$ \\
\hline
\end{tabular}

* Indicates statistically different from referent group $(\mathrm{p}<0.05)$ 
Table 4. Percentage of cancer survivors with low or sufficient strength by years from cancer diagnosis

\begin{tabular}{ccc}
\hline Variable & Low Strength $(<33$ percentile $)$ Sufficient Strength $(\geq 3$ 3rd percentile $)$ \\
\hline 0 years & $8.4 \%(1.8)$ & $9.5 \%(1.7)$ \\
1 year & $13.3 \%(3.0)$ & $7.7 \%(1.7)$ \\
2 years & $6.6 \%(1.6)$ & $7.7 \%(1.9)$ \\
3 years & $10.2 \%(2.2)$ & $4.7 \%(1.0)$ \\
4 years & $2.6 \%(1.0)$ & $5.4 \%(1.4)$ \\
5 years & $4.8 \%(1.0)$ & $7.5 \%(1.5)$ \\
$6+$ years & $54.1 \%(4.6)$ & $57.5 \%(3.5)$ \\
\hline
\end{tabular}

No statistically significant trends between low and sufficient strength $(p>0.05)$ 
Table 5. Mean relative grip strength z-scores by cancer type

\begin{tabular}{lcc}
\multicolumn{1}{c}{ Variable } & Mean (SE) & Confidence Interval \\
\hline Skin Cancers & $0.55(0.14)$ & $0.27-0.84$ \\
$\begin{array}{l}\text { Reproductive Cancers } \\
\begin{array}{l}\text { Digestive, Nervous, Respiratory, } \\
\text { Musculoskeletal, Renal, and } \\
\text { Endocrine }\end{array}\end{array}$ & $0.49(0.12)$ & $0.26-0.74$ \\
Other and Multiple & $0.49(0.10)$ & $0.28-0.70$ \\
\hline
\end{tabular}

None of the four groups are statistically significant $(\mathrm{p}<0.05)$ 


\section{CHAPTER V: EXTENDED LITERATURE REVIEW}

Patients who have been diagnosed with cancer often experience an involuntary loss of weight, particularly from a loss of muscle mass, defined as cachexia ${ }^{10}$. Cancer cachexia affects quality of life (QoL), responsiveness to cancer treatments, and cancer survival negatively ${ }^{4}$. While the scientific understanding of cachexia in the recent years has made many improvements, cancer cachexia persists as an unmet medical need and efforts have been unsuccessful in solidifying a standard treatment guideline ${ }^{4}$.

Numerous studies over the recent 35 years have reported that $50-80 \%$ of cancer survivors are affected by involuntary weight loss; the degree of weight loss is dependent on the tumor type, size, and stage of the cancer ${ }^{9}$. Knowing this, Ryan et al. ${ }^{9}$ explored the effects of weight loss and sarcopenia in response to chemotherapy, quality of life, and survival in patients with cancer. Recent data suggest that weight loss even as small as $2.4 \%$ predicts survival. This is independent of the site or stage of cancer and their performance score. The significance of weight loss on overall survival (OS) has been recognized for years, but more recent use of gold standard methods of body composition has contributed to an increased understanding of the importance specifically of muscle abnormalities. The gold standard method of computer tomography (CT scans) has shown that muscle abnormalities are highly prevalent ranging from $10 \%$ up to $90 \%$. Low muscle mass, also known as Sarcopenia, and low muscle attenuation are the two most prevalent examples of muscle abnormalities. These are essential prognostic indicators of unfavorable outcomes in cancer patients. Both low muscle mass and low muscle attenuation have been associated with significant deterioration in a patients' performance status, an increased risk of postoperative complications, poorer tolerance to chemotherapy, and poorer psychological well-being, overall quality of life, and survival. 
In 2018, Versteeg KS et al. ${ }^{13}$ also conducted a study to investigate whether muscle mass and strength were associated with OS, but specifically in patients with advanced cancer, aged 60 years or older. Before starting chemotherapy, CT scans were used to assess muscle mass and radiodensity, and a hydraulic hand grip dynamometer was used to assess muscle strength. There were 103 participants, with a mean age of 70 years, and were diagnosed with either colorectal, prostate, or breast cancer. The researchers found that, in older patients with advanced cancer, higher muscle strength at the start of chemotherapy is associated with significantly better OS (hazard ratio 1.03; 95\% confidence interval 1.00-1.05). These results suggest that muscle strength could be helpful in estimating cancer survival and could have a large impact on treatment plans. Further research is needed to evaluate more specifically how muscle strength can be used for treatment decisions.

A few years earlier in 2016 Deisenroth A. et al. ${ }^{11}$ also looked at muscle strength and QoL, but specifically in cancer patients who were children in the early phase of primary treatment. In children, muscle strength is crucial for developing motor performance and has an impact on the child's activities of daily living. In this study, 40 children and adolescents with various types of cancer volunteered. The mean age of the participants was 11.39 years \pm 4.08 years. The researchers tested strength performance in a total of 7 muscle groups; the assessments included shoulder abduction, elbow flexor/extensor, hip flexors, knee flexor/extensor, and dorsi-/plantar flexors of the ankle. The participants also completed KINDL questionnaires, which evaluated the patients QoL. Compared with age and gender matched controls, individuals with childhood cancer showed significantly lower strength values in all muscle groups $(\mathrm{p}<0.1)$. The muscles that were most affected were the lower extremities, with a score of $-57.1 \% \pm 10.4 \%$ (median: $-59.2 \%$, minimum: $-75.4 \%$, maximum: $-41.4 \%$ ) percentage deviation from their healthy peers. The children and their 
parents assessed the total QoL to be significantly below age and gender matched reference values as well $(\mathrm{P}<.01)$. This study shows that muscle weakness and decreased $\mathrm{QoL}$ in children and adolescents seem to persist already at the beginning of cancer treatments. This underlines the need of interventions, like exercise programs, to counteract these negative effects starting as early as possible during the treatment process.

As previously stated above, muscle dysfunction and sarcopenia have been associated with greater side effects, poor performance status, and an increased mortality risk in cancer patients. However, insufficient research has examined how performance is affected by specific cancer therapies. In 2017, Klassen O et al. ${ }^{12}$ investigated muscle strength in breast cancer patients in different adjuvant treatment settings. They also compared it with data from healthy individuals. The participants were 255 breast cancer patients from two randomized controlled exercise trials, staged $0-$ III, and aged 54.4 years \pm 9.4 years. The subjects were categorized into four groups according to their treatment status. In addition, another 26 healthy women, aged 53.3 years \pm 9.8 years, were also tested. The researchers assessed muscle function bilaterally through maximal voluntary isometric contractions (MVIC) and maximal isokinetic peak torque (MIPT) by isokinetic dynamometry in shoulder rotators and knee flexors and extensors. They also evaluated shoulder flexibility and muscular fatigue index (FI\%). Lower muscle strength was measured consistently in knee and shoulder strength in subjects after chemotherapy. Patients with cancer had on average up to $12-16 \%$ in upper extremities and $25 \%$ lower strength in lower extremities in MVIC and MIPT compared with healthy women. Patients with radical mastectomy had significantly lower shoulder flexibility, and patients with chemotherapy treatments had consistently higher FI\%. Before and after treatments, breast cancer patients displayed impaired joint dysfunctions and muscle strength. Similar conclusions as the last study were drawn upon in 
this study, as significant differences between patients and healthy individuals highlight the need of exercise therapy as early as possible in order to prevent and counteract the loss of muscle function after treatments, in this case neo/adjuvant chemotherapy and surgery.

Another study done in women diagnosed with breast cancer was done by Perez CS et al. ${ }^{18}$ in 2018. The researchers looked specifically at electromyography of the upper limbs, handgrip strength, and their relationships with dominance in women who have undergone surgery as a treatment for breast cancer. The study evaluated 28 women by measuring strength in their descending trapezius, biceps brachial, triceps brachial, extensor carpi ulnaris, radial extensor carpi and superficial flexor of wrist and fingers using electromyography; they also assessed grip strength using a dynamometer. On the side affected by surgery, the researchers found a significant decrease in hand grip strength and electromyography. However, grip strength showed greater differences when the surgery was performed on the patients' non-dominant side. This shows the need to consider the specifics of treatment, in this case the side affected by surgery and their side of dominance.

While breast cancer is the most common type of cancer among females, prostate cancer is one of the most common types among males according to the American Institute for Cancer Research $^{7}$. In 2015, Gonzalez BD et al. examined changes in muscle strength and physical functioning in men receiving androgen deprivation therapy (ADT) for prostate cancer ${ }^{19}$. The researchers assessed lower body strength through the Chair Rise Test, upper body strength through Handgrip Dynamometry, and measured physical functioning using the SF-12 Physical Functioning self-reported scale. First baseline measures were recorded before ADT began, again after 6 months, and last after 12 months. Physical functioning remained stable in prostate cancer controls, but upper body muscle strength declined in the patients receiving ADT. Lower body strength in 
ADT group was lower than the control group, but lower body strength remained stable. The researchers conclude that this information should be included in the education of risk and benefits associated with ADT, and that further research needs to be done to find ways to prevent or reverse declines in this patient population.

A less common cancer known as Hodgkin lymphoma impacted an estimated 215,531 people in the United States in 2017. In the same year, researcher de Lima FD et al. ${ }^{20}$ wanted to assess fatigue, body composition, strength, muscle quality, and muscle thickness in Hodgkin's lymphoma survivors (HLS). The purpose of this was to compare them to apparently healthy subjects that were matched by gender, age, and physical activity levels. They assessed fatigue using the 20-item Multidimensional Fatigue Inventory. Body composition was assessed with dualenergy x-ray absorptiometry. They measured muscle strength an isokinetic dynamometer. Muscle quality and thickness was measured through B-mode ultrasound. In this study, they discovered that fatigue was significantly higher in HLS than in the healthy control group. Outside of this, there were no significant differences observed. The researchers concluded that potential fatigue should be considered when prescribing exercise training to this specific cancer population.

Another specific cancer type is esophageal. In 2016, Inoue T et al. ${ }^{21}$ sought to evaluate the impact of radial esophagectomy on functional exercise capacity, muscle strength, anxiety, depression, and health related QoL. This was done through a retrospective review of 34 patients with newly diagnosed esophageal cancer, who underwent esophagectomy, and completed postoperative rehabilitation. Hand grip strength, a 6-minute walk test, knee extensor muscle strength, Chronic Obstructive Pulmonary Disease assessment test, and Hospital Anxiety and Depressional Scale were all used to assess the cancer patients. These assessments were completed before the surgery and two weeks post-surgery. All physical status was significantly poorer in 
patients with COPD than those without. There were significant decreases in hand grip strength, the walk test, and knee extensor muscle strength between preoperative and postoperative. The researchers concluded that these results indicate that surgery can be detrimental to patients' health outcomes at two weeks. They also suggest further research should investigate whether prehabilitation would improve postoperative outcomes, QoL, and/or physical fitness.

Cancer cachexia, as stated earlier, has become understood greater in recent years, however it still remains an unmet medical need, and standards for exercise have yet to be decided upon. With the goal to try and help inform the design and duration of physical activity interventions applicable to cancer patients with cachexia, Gale N et al. ${ }^{10}$ teamed up in 2019 to research the change in muscle strength and function over 8 weeks in patients with already established cancer cachexia. They assessed isometric quadriceps and hamstring strength, handgrip strength, standing balance, 10-m walk time, and timed up and go. The patients completed these assessments at baseline, 4 weeks, and 8 weeks. Over the course of the 8 weeks there was little change in performance. The authors consider their study to be exploratory in nature, with challenges such as a small sample size and resultant limitations in interpreting the data. A longer longitudinal study and a larger sample size may aid in more beneficial findings.

Similarly to guidelines concerning cachexia, there are no clear guidelines concerning which type of exercise and which training dose are most effective in cancer patients specifically receiving chemotherapy. In 2016, Van Moll CC et al. ${ }^{22}$ conducted a review to gain insight into the different training modalities during chemotherapy and the effects of training to improve endurance capacity and muscle strength. The researchers wanted to obtain the knowledge to compose a future training program that most effectively trains cancer patients. They began with 809 randomized controlled trials but only 14 met the inclusion criteria. Of these, the authors concluded that the 
studies were poor because the populations varied considerably, and the training programs were very heterogeneous. All in all, more research is needed to find which intensity and duration is most effective for cancer patients. Once this is more developed, hopefully international guidelines can be implemented and put into daily practice.

The next article also looks at cancer relative to exercise. Dankel SJ et al. ${ }^{6}$ knew that skeletal muscle strength and engagement in muscle strengthening activities are each inversely associated with all-cause mortality, but they wanted to know more about their relationship with cancerspecific mortality. In 2018, the researchers used the National Health and Nutrition Examination Survey to assess 2773 individuals aged 50 or older. After adjusting for covariates, individuals in the upper quartile for muscle strength were at a 50\% reduced risk of cancer specific mortality (hazard ratio $=0.50 ; 95 \%$ confidence interval, $0.29-0.85 ; \mathrm{P}=.01$ ) and those meeting muscle strengthening activities were at an $8 \%$ reduced risk of cancer specific mortality, however, this is not statistically significant (hazard ratio $=0.92 ; 95 \%$ confidence interval, $0.45-1.86, \mathrm{P}=.81$ ). They concluded that lower extremity strength should be routinely assessed and engagement in musclestrengthening activities aimed at increasing muscle strength should be promoted.

Another study that looks at the impact of exercise on cancer mortality was conducted in 2017 by Cormie P. et al. ${ }^{5}$; however, this study also aims to look at the impact on cancer recurrence and treatment-related adverse effects. This systematic review comprehensively summarized the available randomized control trial and epidemiologic evidence that investigated the role of exercise in the management of cancer. The authors gathered 100 studies involving thousands of patients whose exercise behavior was assessed following the diagnosis of any type of cancer. In comparison with patients who performed no or less exercise, patients who exercised, following a diagnosis of cancer, were observed to experience fewer or less adverse effects and have a lower relative risk of 
cancer mortality and recurrence. These findings support the stance that exercise should be supplemented in cancer management.

One final systematic review that examines physical activity in cancer prevention and survival was comprised of 45 reports and was published in 2019, authored by McTiernan A. et al. ${ }^{7}$ The article found strong evidence for an association between reduced risk of bladder, breast, colon, endometrial, esophageal, renal, and gastric cancers and highest versus lowest physical activity levels. Relative risk reductions ranged from approximately 10-20\%. They also found moderate associations between decreased all cause and cancer specific mortality and greater amounts of physical activity in individuals with a diagnosis of breast, colorectal, or prostate cancer, with up to a 40-50\% risk reduction. Levels of physical activity recommended in the 2018 Physical Activity Guidelines Advisory Committee Scientific Report are associated with improved survival and risk reduction for several cancers. Further research should explore dose-response relationships and aim to establish mechanisms to explain these associations.

\section{Summary}

Cancer cachexia, loss of muscle mass, negatively affects responsiveness to cancer treatments, quality of life (QoL), and cancer survival. Many patients experience weight loss, which was found to be largely in part of sarcopenia, low muscle mass, and low muscle attenuation. These muscle abnormalities have been associated with significant deterioration in a patients' performance status, an increased risk of postoperative complications, poorer tolerance to chemotherapy, and poorer psychological well-being, overall quality of life, and survival. The literature suggests this is true for older patients aged 60 years or older, children from the very beginning of their treatments, individuals diagnosed with Hodgkin lymphoma, breast, prostate, esophageal, bladder, colon, endometrial, renal, and gastric cancers. There are currently no 
guidelines concerning cachexia or which type of exercise (type and dose) are most effective.

However, this research does show that muscular strength is an important factor in survival and reduces adverse effects. After reviewing the above studies, it is clear muscle strengthening activities should be incorporated into the treatments of cancer patients. 


\section{REFERENCES}

American Cancer Society. Cancer Facts and Figures 2020. Published online September 2020. https://www.cancer.org/content/dam/cancer-org/research/cancer-facts-and-statistics/annualcancer-facts-and-figures/2020/cancer-facts-and-figures-2020.pdf

Gale N, Wasley D, Roberts S, et al. A longitudinal study of muscle strength and function in patients with cancer cachexia. Support Care Cancer Off J Multinatl Assoc Support Care Cancer. 2019;27(1):131-137. doi:10.1007/s00520-018-4297-8

Baker Rogers J, Syed K, Minteer JF. Cachexia. In: StatPearls. StatPearls Publishing; 2020. Accessed February 8, 2021. http://www.ncbi.nlm.nih.gov/books/NBK470208/

Sadeghi M, Keshavarz-Fathi M, Baracos V, Arends J, Mahmoudi M, Rezaei N. Cancer cachexia: Diagnosis, assessment, and treatment. Crit Rev Oncol Hematol. 2018;127:91-104. doi:10.1016/j.critrevonc.2018.05.006

Cormie P, Zopf EM, Zhang X, Schmitz KH. The Impact of Exercise on Cancer Mortality, Recurrence, and Treatment-Related Adverse Effects. Epidemiol Rev. 2017;39(1):71-92. doi:10.1093/epirev/mxx007

Dankel SJ, Loenneke JP, Loprinzi PD. Cancer-Specific Mortality Relative to Engagement in Muscle-Strengthening Activities and Lower Extremity Strength. J Phys Act Health. 2018;15(2):144-149. doi:10.1123/jpah.2016-0204

McTiernan A, Friedenreich CM, Katzmarzyk PT, et al. Physical Activity in Cancer Prevention and Survival: A Systematic Review. Med Sci Sports Exerc. 2019;51(6):1252-1261. doi:10.1249/MSS.0000000000001937 
Ibrahim K, May C, Patel HP, Baxter M, Sayer AA, Roberts H. A feasibility study of implementing grip strength measurement into routine hospital practice (GRImP): study protocol. Pilot Feasibility Stud. 2016;2:27. doi:10.1186/s40814-016-0067-x

Ryan AM, Prado CM, Sullivan ES, Power DG, Daly LE. Effects of weight loss and sarcopenia on response to chemotherapy, quality of life, and survival. Nutr Burbank Los Angel Cty Calif. 2019;67-68:110539. doi:10.1016/j.nut.2019.06.020

Gale N, Wasley D, Roberts S, et al. A longitudinal study of muscle strength and function in patients with cancer cachexia. Support Care Cancer Off J Multinatl Assoc Support Care Cancer. 2019;27(1):131-137. doi:10.1007/s00520-018-4297-8

Deisenroth A, Söntgerath R, Schuster AJ, et al. Muscle strength and quality of life in patients with childhood cancer at early phase of primary treatment. Pediatr Hematol Oncol. 2016;33(6):393-407. doi:10.1080/08880018.2016.1219796

Klassen O, Schmidt ME, Ulrich CM, et al. Muscle strength in breast cancer patients receiving different treatment regimes. J Cachexia Sarcopenia Muscle. 2017;8(2):305-316. doi:10.1002/jcsm.12165

Versteeg KS, Blauwhoff-Buskermolen S, Buffart LM, et al. Higher Muscle Strength Is Associated with Prolonged Survival in Older Patients with Advanced Cancer. The Oncologist. 2018;23(5):580-585. doi:10.1634/theoncologist.2017-0193

Cella DF, Orav EJ, Kornblith AB, et al. Socioeconomic status and cancer survival. J Clin Oncol. 1991;9(8):1500-1509. doi:10.1200/JCO.1991.9.8.1500 
Afshar N, English DR, Blakely T, et al. Differences in cancer survival by area-level socioeconomic disadvantage: A population-based study using cancer registry data. Hsieh JC-H, ed. PLOS ONE. 2020;15(1):e0228551. doi:10.1371/journal.pone.0228551

Why some cancers come back. Published online July 6, 2020.

https://www.cancerresearchuk.org/about-cancer/what-is-cancer/why-some-cancers-comeback

SEER Cancer Statistics Factsheets: Common Cancer Sites. National Cancer Institute. National Cancer Institute https://seer.cancer.gov/statfacts/html/disparities.html

Perez CS, das Neves LMS, Vacari AL, de Cássia Registro Fonseca M, de Jesus Guirro RR, de Oliveira Guirro EC. Reduction in handgrip strength and electromyographic activity in women with breast cancer. J Back Musculoskelet Rehabil. 2018;31(3):447-452. doi:10.3233/BMR-170848

Gonzalez BD, Jim HSL, Small BJ, et al. Changes in physical functioning and muscle strength in men receiving androgen deprivation therapy for prostate cancer: a controlled comparison. Support Care Cancer. 2016;24(5):2201-2207. doi:10.1007/s00520-015-3016-y

de Lima FD, Bottaro M, de Oliveira Valeriano R, et al. Cancer-Related Fatigue and Muscle Quality in Hodgkin's Lymphoma Survivors. Integr Cancer Ther. 2018;17(2):299-305. doi:10.1177/1534735417712009

Inoue $\mathrm{T}$, Ito $\mathrm{S}$, Ando $\mathrm{M}$, et al. Changes in exercise capacity, muscle strength, and health-related quality of life in esophageal cancer patients undergoing esophagectomy. BMC Sports Sci Med Rehabil. 2016;8:34. doi:10.1186/s13102-016-0060-y 
Van Moll CCA, Schep G, Vreugdenhil A, Savelberg HHCM, Husson O. The effect of training during treatment with chemotherapy on muscle strength and endurance capacity: A systematic review. Acta Oncol Stockh Swed. 2016;55(5):539-546. doi:10.3109/0284186X.2015.1127414 